International Electronic Journal of Geometry

Volume 7 No. 2 PP. 7-17 (2014) CIEJG

\title{
PARACOMPLEX LIGHTLIKE SUBMANIFOLDS OF ALMOST PARAHERMITIAN MANIFOLDS
}

\author{
KOUHEI MIURA
}

(Communicated by Kazım İLARSLAN)

\begin{abstract}
We prove that if an almost parahermitian structure of the ambient space induces the identity endomorphism of the radical distribution on a paracomplex lightlike submanifold, then there exists the global lightlike transversal bundle, which is uniquely determined with respect to a screen distribution and a screen transversal bundle of the lightlike submanifold. As an application, we give a sufficient condition that paracomplex lightlike submanifolds in a parakähler manifold are minimal.
\end{abstract}

\section{INTRODUCTION}

Let $M$ be a submanifold in a semi-Riemannian manifold $(\widetilde{M}, \widetilde{g})$. Let $g$ be the induced symmetric (0,2)-tensor field on $M$ from $\widetilde{g}$. Then if the intersection $\operatorname{Rad}(T M)$ of the tangent bundle $T M$ and normal bundle $T M^{\perp}$ of $M$ is a smooth $r$-dimensional distribution, then $(M, g)$ is called an $r$-lightlike submanifold in $(\widetilde{M}, \widetilde{g})$. The distribution $\operatorname{Rad}(T M)$ is called the radical distribution of $(M, g)$. We note that 0 -lightlike submanifolds are semi-Riemannian. The geometry of $r$-lightlike submanifolds with $r>0$ is much different from that of semi-Riemannian submanifolds.

In the case of $r>0$, we can take a semi-Riemannian complementary distribution $S(T M)$ (resp. vector bundle $S\left(T M^{\perp}\right)$ ) of $\operatorname{Rad}(T M)$ in $T M$ (resp. $T M^{\perp}$ ), which is not uniquely determined in general. The fixed distribution $S(T M)$ (resp. vector bundle $S\left(T M^{\perp}\right)$ ) is called the screen distribution (resp. screen transversal vector bundle). Following Duggal and Bejancu [3], we can take a lightlike transversal bundle $\operatorname{ltr}(T M)$ on an open subset $U$ of $M$, which depends on $S(T M), S\left(T M^{\perp}\right)$ and a local basis $\xi=\left(\xi_{1}, \ldots, \xi_{r}\right)$ of $\operatorname{Rad}(T M)$ on $U$. In general, $\operatorname{ltr}(T M)$ is locally constructed on $M$. Then we obtain the decomposition $\left.T \widetilde{M}\right|_{U}=\left.T M\right|_{U} \oplus \operatorname{tr}(T M)$, where $\operatorname{tr}(T M):=S\left(T M^{\perp}\right) \oplus \operatorname{ltr}(T M)$ is called a transversal bundle. The theory of lightlike submanifolds is to study properties of $(M, g)$ which are independent of $S(T M)$ and $S\left(T M^{\perp}\right)$, using the decomposition above. As one of such properties,

Date: Received: November 17, 2013 and Accepted: June 27, 2014.

2010 Mathematics Subject Classification. Primary 53C40; Secondary 53C15.

Key words and phrases. Lightlike submanifold, paracomplex, parahermitian, parakähler, minimal. 
Sakaki introduced the notion of minimal lightlike submanifolds in [5], modifying the definition of minimal lightlike submanifolds in Bejan and Duggal [2].

In this paper, we study a paracomplex lightlike submanifold $M$ of an almost parahermitian manifold $(\widetilde{M}, \widetilde{g}, \widetilde{J})$. Let $J$ be the induced endomorphism of $T M$ from $\widetilde{J}$. In Section 2, we give notions and results we need from paracomplex geometry, and recall some basic facts on lightlike submanifolds following [3]. We prove in Section 3 that if $\left.J\right|_{\operatorname{Rad}(T M)}= \pm I_{\operatorname{Rad}(T M)}$, then there exists the uniquely and globally determined lightlike transversal vector bundle $\operatorname{ltr}(T M)$ with respect to fixed $S(T M)$ and $S\left(T M^{\perp}\right)$. As an application, in Section 4, we can see that co-isotropic paracomplex lightlike submanifolds with $\left.J\right|_{\operatorname{Rad}(T M)}= \pm I_{\operatorname{Rad}(T M)}$ in a parakähler manifold are minimal in the sense of Sakaki [5].

\section{Preliminaries}

In this paper, we assume that all manifolds are connected, paracompact and differentiable of class $C^{\infty}$. Let $E$ be a vector bundle over a manifold $N$ and $\operatorname{End}(E)$ be the vector bundle of which the fiber on $p \in N$ is End $\left(E_{p}\right)$. The identity endomorphism of $E$ is denoted by $I_{E}$. We denote the algebra of smooth functions on $N$ by $\mathcal{F}(N)$, and the $\mathcal{F}(N)$-module of smooth sections of $E$ by $\Gamma(E)$. For a section $P \in \Gamma($ End $(E))$ and a linear connection $\nabla^{E}$ of $E$, we define the differential $\nabla^{E} P$ of $P$ with respect to $\nabla^{E}$ by

$$
\left(\nabla_{X}^{E} P\right)(s):=\nabla_{X}^{E}(P(s))-P\left(\nabla_{X}^{E} s\right), \quad \forall X \in \Gamma(T N), \forall s \in \Gamma(E),
$$

where $T N$ is the tangent bundle of $N$.

An endomorphism $\widetilde{J} \in$ End $(T \widetilde{M})$ is an almost product structure of a manifold $\widetilde{M}$, if $\widetilde{J}^{2}=I_{T \widetilde{M}}$ and $\widetilde{J} \neq \pm I_{T \widetilde{M}}$. If there exists a nondegenerate metric on $\widetilde{M}$ such that

$$
\widetilde{g}(\widetilde{J} X, \widetilde{J} Y)=-\widetilde{g}(X, Y), \quad \forall X, Y \in \Gamma(T \widetilde{M}),
$$

then we say that $(\widetilde{M}, \widetilde{g}, \widetilde{J})$ is an almost parahermitian manifold. If $\widetilde{J}$ is integrable, we say that $(\widetilde{M}, \widetilde{g}, \widetilde{J})$ is a parahermitian manifold. We note that $\widetilde{J}$ is integrable, if and only if the Nijenhuis tensor $N$ of $\widetilde{J}$ given by

$$
N(X, Y):=[\widetilde{J} X, \widetilde{J} Y]-\widetilde{J}[\widetilde{J} X, Y]-\widetilde{J}[X, \widetilde{J} Y]+[X, Y]
$$

vanishes identically on $\widetilde{M}$. For a torsion-free affine connection $\hat{\nabla}$ on $\widetilde{M}$, putting $(\hat{\nabla} \widetilde{J})(X, Y):=\left(\hat{\nabla}_{X} \widetilde{J}\right)(Y)$ for any $X, Y \in T \widetilde{M}$, we obtain

$$
N(X, Y)=(\hat{\nabla} \widetilde{J})(\widetilde{J} X, Y)-(\hat{\nabla} \widetilde{J})(\widetilde{J} Y, X)+\widetilde{J}(\hat{\nabla} \widetilde{J})(Y, X)-\widetilde{J}(\hat{\nabla} \widetilde{J})(X, Y) .
$$

We say that an almost parahermitian manifold $(\widetilde{M}, \widetilde{g}, \widetilde{J})$ is a parakählerian manifold if $\widetilde{J}$ is parallel with respect to the Levi-Civita connection $\widetilde{\nabla}$ of the semi-Riemannian manifold $(\widetilde{M}, \widetilde{g}):\left(\widetilde{\nabla}_{X} \widetilde{J}\right)(Y)=0$ for any $X, Y \in T \widetilde{M}$. Thus, if $(\widetilde{M}, \widetilde{J}, \widetilde{g})$ is parakählerian, then $\widetilde{J}$ is integrable.

Let $\left(E, g^{E}\right)$ be a semi-Riemannian vector bundle over a manifold $M$, that is, $g^{E}$ is a nondegenerate bundle metric of $E$. An endomorphism $J^{E} \in \operatorname{End}(E)$ is a parahermitian structure, if $\left(J^{E}\right)^{2}=I_{E}$ and $g^{E}\left(J^{E} s_{1}, J^{E} s_{2}\right)=-g^{E}\left(s_{1}, s_{2}\right)$ for any $s_{1}, s_{2} \in \Gamma(E)$. The triplet $\left(E, g^{E}, J^{E}\right)$ is called a parahermitian vector bundle. We note that the rank of a parahermitian vector bundle is even and the metric is neutral. Thus, it follows that any almost parahermitian manifold $(\widetilde{M}, \widetilde{g}, \widetilde{J})$ has even 
dimension, say $2 m$, and the index ind $\widetilde{M}$ is equal to $m$. Thus $(\widetilde{M}, \widetilde{g})$ is a neutral semi-Riemannian manifold.

Putting $E^{ \pm}:=\operatorname{Ker}\left(J^{E} \mp I_{E}\right)$ for a parahermitian vector bundle $\left(E, g^{E}, J^{E}\right)$ with rank $2 k$, we obtain the non-orthogonal direct decomposition $E=E^{+} \oplus E^{-}$, where $E^{ \pm}$is the eigenspace of $J^{E}$ corresponding to the eigenvalue \pm 1 . Then $\operatorname{rank} E^{ \pm}=k$ and the subbundles are totally lightlike (isotropic), that is, $g^{E}$ vanishes on each of them. Then we denote the $E^{ \pm}$-component of $s \in E$ by $s^{ \pm}$.

A tangent vector $v$ of $(\widetilde{M}, \widetilde{g})$ is said to be spacelike, timelike, or null according as we have $v=0$ or $\widetilde{g}(v, v)>0, \widetilde{g}(v, v)<0$, or $\widetilde{g}(v, v)=0$ and $v \neq 0$. It is easy that $\widetilde{J}(v)$ is perpendicular to $v$ for any $v \in T \widetilde{M}$, and $\widetilde{J}(v)$ is timelike (resp. spacelike) for any spacelike (resp. timelike) tangent vector $v \in T \widetilde{M}$.

We recall some basic results on lightlike submanifolds of a semi-Riemannian manifold. With respect to this class of submanifolds, we refer the monograph by Duggal and Bejancu [3]. (See Duggal and Sahin [4] also.)

Let $(\widetilde{M}, \widetilde{g})$ be an $n$-dimensional semi-Riemannian manifold with index $t$. An $m$-dimensional submanifold $M$ of $(\widetilde{M}, \widetilde{g})$ is said to be $r$-lightlike if the subset of $T M$ :

$$
\operatorname{Rad}(T M):=\bigcup_{p \in M} \operatorname{Rad}\left(T_{p} M\right), \quad \text { where } \operatorname{Rad}\left(T_{p} M\right):=T_{p} M \cap T_{p} M^{\perp},
$$

is a smooth distribution on $M$ of rank $r$ called the lightlike distribution. Then we see that the rank $r$ of $\operatorname{Rad}(T M)$ satisfies

$$
r \leq \min \{t, n-t, m, n-m\} .
$$

It follows that $M$ is $r$-lightlike if and only if the induced tensor field $g$ on $M$ by $\widetilde{g}$ has a constant rank $m-r$. In the case of $r=0$, i.e., the distribution $\operatorname{Rad}(T M)$ is zero, $(M, g)$ is a semi-Riemannian submanifold.

By the definition above, we see that the normal bundle $T M^{\perp}$ of $M$ is not complementary to $T M$ in $T \widetilde{M}$ along $M$ if $r>0$. Then we take two vector bundles $S(T M)$ and $S\left(T M^{\perp}\right)$, whose existences are consequences of the paracompactness of $M$, such that

$$
T M=S(T M) \oplus_{\text {orth }} \operatorname{Rad}(T M) \text { and } T M^{\perp}=\operatorname{Rad}(T M) \oplus_{\text {orth }} S\left(T M^{\perp}\right),
$$

which $\oplus_{\text {orth }}$ stands for orthogonal direct sum of vector bundles. We call $S(T M)$ and $S\left(T M^{\perp}\right)$ a screen distribution and a screen transversal vector bundle of $M$, respectively. We note that both $S(T M)$ and $S\left(T M^{\perp}\right)$ are nondegenerate vector subbundles of $T \widetilde{M}$ along $M$.

For a fixed screen distribution $S(T M)$, we can take the complementary orthogonal vector subbundle $S(T M)^{\perp}$ in $T \widetilde{M}$ along $M$ :

$$
\left.T \widetilde{M}\right|_{M}=S(T M) \oplus_{\text {orth }} S(T M)^{\perp},
$$

which is automatically nondegenerate. Since another fixed object $S\left(T M^{\perp}\right)$ is a vector subbundle of $S(T M)^{\perp}$, we can take the complementary orthogonal vector subbundle $S\left(T M^{\perp}\right)^{\perp}$ in $S(T M)^{\perp}$ such that

$$
S(T M)^{\perp}=S\left(T M^{\perp}\right) \oplus_{\text {orth }} S\left(T M^{\perp}\right)^{\perp} .
$$

We note that $\operatorname{Rad} T M \subset S\left(T M^{\perp}\right)^{\perp}$. 
For a local basis $\xi=\left(\xi_{1}, \ldots, \xi_{r}\right)$ of $\operatorname{Rad}(T M)$ on an open subset $U$ of $M$, we can take local sections $N_{1}, \ldots, N_{r}$ of $S\left(T M^{\perp}\right)^{\perp}$ on $U$ such that

$$
\widetilde{g}\left(\xi_{i}, N_{j}\right)=\delta_{i j} \quad \text { and } \quad \widetilde{g}\left(N_{i}, N_{j}\right)=0 \text { for any } i, j=1,2, \ldots, r .
$$

Then we obtain a complementary vector bundle $\operatorname{ltr}(T M):=\operatorname{Span}\left\{N_{1}, \ldots, N_{r}\right\}$ to $\operatorname{Rad}(T M)$ in $S\left(T M^{\perp}\right)^{\perp}$ on $U$ (cf. [3]). We call $\operatorname{ltr}(T M)$ the lightlike transversal bundle. This enables us to consider the vector bundle:

$$
\operatorname{tr}(T M):=S\left(T M^{\perp}\right) \oplus_{\text {orth }} \operatorname{ltr}(T M),
$$

which is a complementary vector bundle to $T M$ in $T \widetilde{M}$ along $U \subset M$. We call $\operatorname{tr}(T M)$ the transversal vector bundle. Then we have the following decompositions:

$$
\begin{aligned}
\left.T \widetilde{M}\right|_{U} & =T M \oplus \operatorname{tr}(T M) \\
& =\left(S(T M) \oplus_{\text {orth }} \operatorname{Rad}(T M)\right) \oplus\left(S\left(T M^{\perp}\right) \oplus_{\text {orth }} \operatorname{ltr}(T M)\right) \\
& =S(T M) \oplus_{\text {orth }} S(T M)^{\perp} \oplus_{\text {orth }}(\operatorname{Rad}(T M) \oplus \operatorname{ltr}(T M)),
\end{aligned}
$$

where $\oplus$ stands for non-orthogonal direct sum of vector bundles. We note that $\operatorname{tr}(T M)$ is never orthogonal to $T M$, if $r>0$.

Let $M$ be a submanifold of an almost parahermitian manifold $(\widetilde{M}, \widetilde{g}, \widetilde{J})$. We say that $M$ is a paracomplex submanifold if the tangent space $T_{p} M$ at any point $p$ of $M$ is $\widetilde{J}$-invariant in $T_{p} \widetilde{M}$, that is, $\widetilde{J}\left(T_{p} M\right)=T_{p} M$ for any $p \in M$. Then the normal vector bundle $T M^{\perp}$ is also $\widetilde{J}$-invariant.

For a paracomplex $r$-lightlike submanifold $M$ of $(\widetilde{M}, \widetilde{g}, \widetilde{J}), M$ has the induced symmetric $(0,2)$-tensor field $g$ from $\widetilde{g}$ and the induced endomorphism $J$ from $\widetilde{J}$ on $M$. We note that $J$ is not necessarily $J \neq \pm I_{T M}$.

\section{PARACOMPlEX Lightlike SUbManifolds of ALMOST PARAhERMitian MANIFOLDS}

Let $(\widetilde{M}, \widetilde{g}, \widetilde{J})$ be a $2 n$-dimensional almost parahermitian manifold with index $n$. Let $M$ be an $m$-dimensional paracomplex $r$-lightlike submanifold of $(\widetilde{M}, \widetilde{g}, \widetilde{J})$ and $J$ (resp. $g$ ) the induced endomorphism from $\widetilde{J}$ (resp. symmetric $(0,2)$-tensor field from $\widetilde{g}$ ) on $M$. We note that the dimension $m$ of $M$ is not necessary even, in contrast to the theory of nondegenerate paracomplex submanifolds in almost parahermitian manifolds.

Theorem 3.1. Let $(M, J, g)$ be a paracomplex $r$-lightlike submanifold of an almost parahermitian manifold $(\widetilde{M}, \widetilde{J}, \widetilde{g})$. Then we have the following assertions:

(i) The lightlike distribution $\operatorname{Rad}(T M)$ is J-invariant.

(ii) There exists a J-invariant screen distribution $S(T M)$ on $M$.

(iii) There exists a $\widetilde{J}$-invariant screen transversal bundle $S\left(T M^{\perp}\right)$ on $M$.

Moreover, the induced metrics of $S(T M)$ and $S\left(T M^{\perp}\right)$ are parahermitian. Thus these are neutral.

Proof. (i) Since $T M$ and $T M^{\perp}$ are $\widetilde{J}$-invariant, the intersection $\operatorname{Rad}(T M):=T M \cap$ $T M^{\perp}$ is also $\widetilde{J}$-invariant in $T M$.

In order to prove (ii) (resp. (iii)), we take a positive definite metric $l$ (resp. $l^{\perp}$ ) of $T M$ (resp. $T M^{\perp}$ ) whose existence is a consequence of the paracompactness of 
M. Put

$$
\begin{aligned}
k(X, Y) & :=l(X, Y)+l(J X, J Y), \\
\left(\operatorname{resp.} \quad k^{\perp}(V, W)\right. & \left.:=l^{\perp}(V, W)+l^{\perp}(\widetilde{J} V, \widetilde{J} W)\right),
\end{aligned}
$$

where $X, Y \in T M$ (resp. $V, W \in T M^{\perp}$ ). Since $T M$ (resp. $T M^{\perp}$ ) is $J$-invariant (resp. $\left.\widetilde{J}_{-}\right), k$ (resp. $k^{\perp}$ ) is also a positive definite metric. We can take as a screen distribution $S(T M)$ (resp. screen transversal bundle $S\left(T M^{\perp}\right)$ ) of $M$ the complementary orthogonal distribution to $\operatorname{Rad}(T M)$ in $T M$ (resp. the complementary orthogonal subbundle to $\operatorname{Rad}(T M)$ in $\left.T M^{\perp}\right)$ with respect to $k$ (resp. $k^{\perp}$ ). It is easy to see that

$$
k(J X, \xi)=k(X, J \xi)=0, \quad k^{\perp}(\widetilde{J} V, \xi)=k^{\perp}(V, \widetilde{J} \xi)=0,
$$

where any $X \in S(T M), \xi \in \operatorname{Rad}(T M), V \in S\left(T M^{\perp}\right)$. Therefore $S(T M)$ (resp. $\left.S\left(T M^{\perp}\right)\right)$ is $J$-invariant (resp. $\widetilde{J}$-invatiant). This completes the proof of our assertion (ii) (resp. (iii)). Since $S(T M)$ (resp. $\left.S\left(T M^{\perp}\right)\right)$ is complementary to $\operatorname{Rad}(T M)$ in $T M$ (resp. $T M^{\perp}$ ), the induced tensor from $\widetilde{g}$ is nondegenerate. In particular, $S(T M)$ and $S\left(T M^{\perp}\right)$ are parahermitian bundles with respect to the induced objects from $\widetilde{g}$ and $\widetilde{J}$.

Remark 3.1. Theorem 3.1 is a generalization of Theorem 4.2 in [1].

By Theorem 3.1, since $S(T M)$ and $S\left(T M^{\perp}\right)$ are parahermitian, the rank of both $S(T M)$ and $S\left(T M^{\perp}\right)$ are even. Thus, we obtain the following corollary:

Corollary 3.1. Let $(M, g, J)$ be a paracomplex $r$-lightlike submanifold of an almost parahermitian manifold $(\widetilde{M}, \widetilde{g}, \widetilde{J})$. If the dimension of $M$ is odd (resp. even), then $r$ is odd (resp. even). Hence, there exist no odd-dimensional paracomplex semiRiemannian submanifolds.

In this paper, we call submanifolds with real codimension one hypersurfaces. From the inequality (2.1), we have

Corollary 3.2. Any paracomplex $r$-lightlike hypersurface $(M, g, J)$ of an almost parahermitian manifold $(\widetilde{M}, \widetilde{g}, \widetilde{J})$ is 1-lightlike.

Remark 3.2. corollary 3.1 and 3.2 are generalizations of Theorem 4.1 in [1]. We note that lightlike submanifolds with real codimension two are called "hypersurfaces" in Section 4 of [1].

Lemma 3.1. Let $(M, g, J)$ be a paracomplex $r$-lightlike submanifold of an almost parahermitian manifold $(\widetilde{M}, \widetilde{g}, \widetilde{J})$. There exists a local basis $\xi=\left(\xi_{1}, \ldots, \xi_{r}\right)$ of $\operatorname{Rad}(T M)$ such that $J\left(\xi_{i}\right)=+\xi_{i}$ or $-\xi_{i}$, that is, $\xi_{i}$ is a local eigensection of $J$.

Proof. For an everywhere nonzero local section $\zeta \in \Gamma(\operatorname{Rad})$ on an open subset $U \subset M$, if $\zeta \wedge J(\zeta)=0$ on $U$, then we take $\xi_{1}:=\zeta$. Otherwise, we can put $\xi_{1}:=\zeta+J(\zeta)$. For $l(1 \leq l<r)$, we assume that $\xi_{1}, \xi_{2}, \ldots, \xi_{l}$ are eigensections which are linearly independent on an open set $U^{\prime} \subset U$, that is, $\xi_{1} \wedge \cdots \wedge \xi_{l} \neq 0$ on $U^{\prime}$. There exists a local section $\zeta \in \Gamma(\operatorname{Rad}(T M))$ such that $\zeta \notin \operatorname{Span}\left\{\xi_{1}, \ldots, \xi_{l}\right\}$ on $U^{\prime}$. If $\zeta \wedge J(\zeta)=0$ on $U^{\prime}$, then we take $\xi_{l+1}:=\zeta$. Otherwise, we put $\xi_{ \pm}:=\zeta \pm J(\zeta)$. 
Then, it follows that $\xi_{+} \notin \operatorname{Span}\left\{\xi_{1}, \ldots, \xi_{l}\right\}$ or $\xi_{-} \notin \operatorname{Span}\left\{\xi_{1}, \ldots, \xi_{l}\right\}$ on $U^{\prime}$. Indeed, in case of $\xi_{+} \in \operatorname{Span}\left\{\xi_{1}, \ldots, \xi_{l}\right\}$, we can see

$$
\begin{aligned}
0 & =\xi_{+} \wedge \xi_{1} \wedge \cdots \wedge \xi_{l} \\
& =\zeta \wedge \xi_{1} \wedge \cdots \wedge \xi_{l}+J(\zeta) \wedge \xi_{1} \wedge \cdots \wedge \xi_{l},
\end{aligned}
$$

therefore, we get $J(\zeta) \wedge \xi_{1} \wedge \cdots \wedge \xi_{l}=-\zeta \wedge \xi_{1} \wedge \cdots \wedge \xi_{l} \neq 0$. Thus, we get

$$
\xi_{-} \wedge \xi_{1} \wedge \cdots \wedge \xi_{l}=2\left(\zeta \wedge \xi_{1} \wedge \cdots \wedge \xi_{l}\right) \neq 0 \text { on } U^{\prime}
$$

So $\xi_{-} \notin \operatorname{Span}\left\{\xi_{1}, \ldots, \xi_{l}\right\}$ on $U^{\prime}$. By the inductively way, we can obtain a required local basis $\xi=\left(\xi_{1}, \ldots, \xi_{r}\right)$ of $\operatorname{Rad}(T M)$.

Theorem 3.2. Let $(M, g, J)$ be a paracomplex $r$-lightlike submanifold of an almost parahermitian manifold $(\widetilde{M}, \widetilde{g}, \widetilde{J})$. For $\left(M, g, J, S(T M), S\left(T M^{\perp}\right)\right)$ and a local basis $\xi=\left(\xi_{1}, \ldots, \xi_{r}\right)$ of $\left.\operatorname{Rad}(T M)\right|_{U}$ as in Lemma 3.1, where $U$ is an open set of $M$, there exist local smooth sections $\eta_{1}, \ldots, \eta_{r}$ of $\left.S\left(T M^{\perp}\right)^{\perp}\right|_{U}$ such that

$$
\widetilde{J}\left(\eta_{i}\right)=-\varepsilon_{i} \eta_{i}, \quad \widetilde{g}\left(\xi_{i}, \eta_{j}\right)=\delta_{i j}, \quad \widetilde{g}\left(\eta_{i}, \eta_{j}\right)=0,
$$

where $\varepsilon_{i} \in\{+1,-1\}$ is an eigenvalue of $\xi_{i}$ for $J$, that is, the signature defined by $J\left(\xi_{i}\right)=\varepsilon_{i} \xi_{i}$, and $i, j \in\{1, \ldots, r\}$.

Proof. By [3], for a local basis $\xi=\left(\xi_{1}, \ldots, \xi_{r}\right)$ of $\operatorname{Rad}(T M)$ on $U \subset M$, we can take local sections $N_{1}, \ldots, N_{r}$ of $S\left(T M^{\perp}\right)^{\perp}$ on $U$ such that

$$
\widetilde{g}\left(\xi_{i}, N_{j}\right)=\delta_{i j}, \quad \widetilde{g}\left(N_{i}, N_{j}\right)=0 \quad \text { for any } i, j \in\{1,2, \ldots, r\} .
$$

We define

$$
\eta_{i}:=\frac{1}{2}\left(N_{i}-\varepsilon_{i} \widetilde{J}\left(N_{i}\right)\right) \text { for } i \in\{1, \ldots, r\} .
$$

It is easy to check $\widetilde{J}\left(\eta_{i}\right)=-\varepsilon_{i} \eta_{i}$ for any $i \in\{1, \ldots, r\}$. Moreover, we have

$$
\begin{aligned}
2 \widetilde{g}\left(\xi_{i}, \eta_{j}\right) & =\widetilde{g}\left(\xi_{i}, N_{j}-\varepsilon_{j} \widetilde{J}\left(N_{j}\right)\right)=\widetilde{g}\left(\xi_{i}, N_{j}\right)-\varepsilon_{j} \widetilde{g}\left(\xi_{i}, \widetilde{J}\left(N_{j}\right)\right) \\
& =\widetilde{g}\left(\xi_{i}, N_{j}\right)+\varepsilon_{j} \widetilde{g}\left(\widetilde{J}\left(\xi_{i}\right), N_{j}\right)=2 \delta_{i j} .
\end{aligned}
$$

Thus $\widetilde{g}\left(\xi_{i}, \eta_{j}\right)=\delta_{i j}$ for any $i, j \in\{1, \ldots, r\}$.

With respect to the local null frame $\xi_{1}, \ldots, \xi_{r}, N_{1}, \ldots, N_{r}$ of $S\left(T M^{\perp}\right)^{\perp}$,

$$
\begin{aligned}
\widetilde{J}\left(N_{i}\right) & =\sum_{j=1}^{r}\left(\widetilde{g}\left(\widetilde{J}\left(N_{i}\right), N_{j}\right) \xi_{j}+\widetilde{g}\left(\widetilde{J}\left(N_{i}\right), \xi_{j}\right) N_{j}\right) \\
& =\sum_{j=1}^{r} \widetilde{g}\left(\widetilde{J}\left(N_{i}\right), N_{j}\right) \xi_{j}-\varepsilon_{i} N_{i} .
\end{aligned}
$$

Applying $\widetilde{J}$ to the above equation, we have

$$
N_{i}=\sum_{j=1}^{r} \varepsilon_{j} \widetilde{g}\left(\widetilde{J}\left(N_{i}\right), N_{j}\right) \xi_{j}-\varepsilon_{i} \widetilde{J}\left(N_{i}\right) .
$$

Substituting (3.3) into (3.2), we obtain

$$
\sum_{j=1}^{r}\left(1-\varepsilon_{i} \varepsilon_{j}\right) \widetilde{g}\left(\widetilde{J}\left(N_{i}\right), N_{j}\right) \xi_{j}=0 .
$$

Consequently we can see

$$
\widetilde{g}\left(\widetilde{J}\left(N_{i}\right), N_{j}\right)=0 \text { for any } i, j \text { such that } \varepsilon_{j}=-\varepsilon_{i} .
$$


On the other hand,

$$
\begin{aligned}
4 \widetilde{g}\left(\eta_{i}, \eta_{j}\right) & =\widetilde{g}\left(N_{i}-\varepsilon_{i} \widetilde{J}\left(N_{i}\right), N_{j}-\varepsilon_{j} \widetilde{J}\left(N_{j}\right)\right) \\
& =\left(\varepsilon_{j}-\varepsilon_{i}\right) \widetilde{g}\left(\widetilde{J}\left(N_{i}\right), N_{j}\right) \\
& = \begin{cases}0 & \text { for any } i, j \text { such that } \varepsilon_{j}=\varepsilon_{i}, \\
2 \varepsilon_{j} \widetilde{g}\left(\widetilde{J}\left(N_{i}\right), N_{j}\right) & \text { for any } i, j \text { such that } \varepsilon_{j}=-\varepsilon_{i} .\end{cases}
\end{aligned}
$$

By the equation above and (3.4), we obtain $\widetilde{g}\left(\eta_{i}, \eta_{j}\right)=0$ for any $i, j \in\{1, \ldots, r\}$. This completes the proof of our assertion.

For a paracomplex $r$-lightlike submanifold $\left(M, g, J, S(T M), S\left(T M^{\perp}\right)\right)$ and a local basis $\xi=\left(\xi_{1}, \ldots, \xi_{r}\right)$ of $\operatorname{Rad}(T M)$ as in Lemma 3.1, by virtue of Theorem 3.2, we can define over $U$ :

$$
\begin{aligned}
\operatorname{ttr}(T M) & :=\operatorname{ltr}(T M, \xi):=\operatorname{Span}\left\{\eta_{1}, \ldots, \eta_{r}\right\}, \\
\operatorname{tr}(T M) & :=\operatorname{tr}(T M, \xi):=S\left(T M^{\perp}\right) \oplus \operatorname{ltr}(T M) .
\end{aligned}
$$

We obtain the following:

Theorem 3.3. Let $(M, g, J)$ be a paracomplex $r$-lightlike submanifold of an almost parahermitian manifold $(\widetilde{M}, \widetilde{g}, \widetilde{J})$. For $\left(M, g, J, S(T M), S\left(T M^{\perp}\right)\right)$ and a basis $\xi=$ $\left(\xi_{1}, \ldots, \xi_{r}\right)$ of $\left.\operatorname{Rad}(T M)\right|_{U}$ as in Lemma 3.1, where $U$ is an open set of $M$, there exist local decompositions of vector bundles over $U$ :

$$
\begin{aligned}
\left.T \widetilde{M}\right|_{U} & =T M \oplus \operatorname{tr}(T M) \\
& =\left(S(T M) \oplus_{\text {orth }} \operatorname{Rad}(T M)\right) \oplus\left(S\left(T M^{\perp}\right) \oplus_{\text {orth }} \operatorname{ltr}(T M)\right) \\
& =S(T M) \oplus_{\text {orth }} S\left(T M^{\perp}\right) \oplus_{\text {orth }}(\operatorname{Rad}(T M) \oplus \operatorname{ltr}(T M)),
\end{aligned}
$$

where $S(T M), S\left(T M^{\perp}\right), \operatorname{Rad}(T M)$ and $\operatorname{ltr}(T M)$ are $\widetilde{J}$-invariant, and $S(T M)$, $S\left(T M^{\perp}\right)$ and $(\operatorname{Rad}(T M) \oplus \operatorname{ltr}(T M))$ are parahermitian vector bundles over $U$.

According to the $\widetilde{J}$-invariant decomposition over $U:\left.T \widetilde{M}\right|_{U}=T M \oplus \operatorname{tr}(T M)$ as in Theorem 3.3, we have the Gauss formula and the Weingarten formula:

$$
\begin{aligned}
& \widetilde{\nabla}_{X} Y=\nabla_{X} Y+h(X, Y), \quad X, Y \in \Gamma(T M), \\
& \widetilde{\nabla}_{X} V=-A_{V} X+\nabla_{X}^{\operatorname{tr}} V, \quad V \in \Gamma(\operatorname{tr}(T M)),
\end{aligned}
$$

where $\nabla_{X} Y$ (resp. $h(X, Y)$ ) is the tangential (resp. transversal) component of $\widetilde{\nabla}_{X} Y$, and $-A_{V} X$ (resp. $\nabla_{X}^{\text {tr }} V$ ) is the tangential (resp. transversal) component of $\widetilde{\nabla}_{X} V$. We note that the induced connection $\nabla$ is not necessary a metric connection in case of $r>0$ and refer details for [3] and [4].

We note that $\left.J\right|_{\operatorname{Rad}(T M)}$ is not necessary $\left.J\right|_{\operatorname{Rad}(T M)} \neq \pm I_{\operatorname{Rad}(T M)}$. We put $k:=\operatorname{rank}\left(\operatorname{Ker}\left(\left.J\right|_{\operatorname{Rad}(T M)}-I_{\operatorname{Rad}(T M)}\right)\right)$. Hereafter we use the induces $i, j, \alpha, \beta$ and $A, B$ for the following range respectively:

$$
i, j=1, \ldots, k ; \quad \alpha, \beta=k+1, \ldots, r ; \quad A, B=1, \ldots, r .
$$

From now on, we take a local basis of $\operatorname{Rad}(T M)$ as in Lemma 3.1 as follows:

$$
\xi=\left(\xi^{+} ; \xi^{-}\right)=\left(\xi_{1}^{+}, \ldots, \xi_{k}^{+} ; \xi_{k+1}^{-}, \ldots, \xi_{r}^{-}\right),
$$


where $\left.J\right|_{\operatorname{Rad}(T M)}\left(\xi_{i}^{+}\right)=\xi_{i}^{+},\left.J\right|_{\operatorname{Rad}(T M)}\left(\xi_{\alpha}^{-}\right)=-\xi_{\alpha}^{-}$. Furthermore, we denote the local basis of $\operatorname{ltr}(T M)$ constructed corresponding to $\xi$ in Theorem 3.2 by

$$
\eta=\left(\eta^{-} ; \eta^{+}\right)=\left(\eta_{1}^{-}, \ldots, \eta_{k}^{-} ; \eta_{k+1}^{+}, \ldots, \eta_{r}^{+}\right) .
$$

It follows that $\left.J\right|_{\operatorname{Rad}(\mathrm{TM})}\left(\eta_{i}^{-}\right)=-\eta_{i}^{-}$and $\left.J\right|_{\operatorname{Rad}(T M)}\left(\eta_{\alpha}^{+}\right)=\eta_{\alpha}^{+}$. We denote the local basis of $S\left(T M^{\perp}\right)^{\perp}=\operatorname{Rad}(T M) \oplus \operatorname{ltr}(T M, \xi)$ by $(\xi ; \eta)$.

Lemma 3.2. Let $\left(M, g, J, S(T M), S\left(T M^{\perp}\right)\right)$ be a paracomplex $r$-lightlike submanifold of an almost parahermitian manifold $(\widetilde{M}, \widetilde{g}, \widetilde{J})$. For local bases $(\xi ; \eta)$ and $\left(\xi^{\prime} ; \eta^{\prime}\right)$ of $S\left(T M^{\perp}\right)^{\perp}$ on $U$ and $U^{\prime}$ respectively, the transition matrix at $p \in U \cap U^{\prime}$ is

$$
\left(\begin{array}{llll}
\xi^{\prime} & \xi^{\prime}- & \eta^{\prime} & \eta^{\prime}
\end{array}\right)_{p}=\left(\begin{array}{lll}
\xi^{+} & \xi^{-} & \eta^{-}
\end{array} \eta^{+}\right)_{p}\left[\begin{array}{cccc}
A_{+} & O & O & B_{+} \\
O & A_{-} & B_{-} & O \\
O & O & C_{-} & O \\
O & O & O & C_{+}
\end{array}\right],
$$

where $A_{+}, C_{-} \in G L_{k}(\mathbb{R}), A_{-}, C_{+} \in G L_{r-k}(\mathbb{R})$ and $B_{+},{ }^{t} B_{-} \in M_{k, r-k}(\mathbb{R})$, and these matrices satisfy

$$
C_{-}={ }^{t} A_{+}^{-1}, \quad C_{+}={ }^{t} A_{-}^{-1}, \quad B_{-}=-A_{-}{ }^{t} B_{+}{ }^{t} A_{+}^{-1} .
$$

Proof. Since $\xi$ and $\xi^{\prime}$ are bases of $\operatorname{Rad}(T M)_{p}$ and eigenvectors of $J$, we obtain

$$
\xi_{j}^{\prime}=\sum_{i=1}^{k} a_{i j} \xi_{i}^{+}, \quad \xi_{\beta}^{\prime-}=\sum_{\alpha=k+1}^{r} a_{\alpha \beta} \xi_{\alpha}^{-},
$$

where $j \in\{1, \ldots, k\}$ and $\beta \in\{k+1, \ldots, r\}$. Then it follows that $A_{+}:=\left(a_{i j}\right) \in$ $G L_{k}(\mathbb{R})$ and $A_{-}:=\left(a_{\alpha \beta}\right) \in G L_{r-k}(\mathbb{R})$. Since $\xi_{A}^{ \pm}, \xi_{A}^{\prime}{ }^{ \pm}, \eta_{A}^{ \pm}$and $\eta_{A}^{\prime \pm}(A \in\{1, \ldots, r\})$ are eigenvectors of $\widetilde{J}$ in $S\left(T M^{\perp}\right)_{p}^{\perp}$, we obtain

$$
\eta_{j}^{\prime-}=\sum_{\alpha=k+1}^{r} b_{\alpha j} \xi_{\alpha}^{-}+\sum_{i=1}^{k} c_{i j} \eta_{i}^{-}, \quad \eta_{\beta}^{\prime}=\sum_{i=1}^{k} b_{i \beta} \xi_{i}^{+}+\sum_{\alpha=k+1}^{r} c_{\alpha \beta} \eta_{\alpha}^{+},
$$

where $j \in\{1, \ldots, k\}$ and $\beta \in\{k+1, \ldots, r\}$. We put $B_{-}:=\left(b_{\alpha j}\right), B_{+}:=\left(b_{i \beta}\right)$, $C_{-}:=\left(c_{i j}\right)$ and $C_{+}:=\left(c_{\alpha \beta}\right)$. From $\widetilde{g}\left(\xi_{i}^{\prime}, \eta_{j}^{\prime}\right)=\delta_{i j}, \widetilde{g}\left(\xi_{\alpha}^{\prime}, \eta_{\beta}^{+}\right)=\delta_{\alpha \beta}$ and $\widetilde{g}\left(\xi_{i}^{\prime}, \eta_{\beta}^{+}\right)=\widetilde{g}\left(\xi_{\alpha}^{\prime}, \eta_{j}^{\prime}\right)=0$,

$$
\delta_{i j}=\widetilde{g}\left(\xi_{i}^{\prime}{ }^{+}, \eta_{j}^{\prime-}\right)=\sum_{l=1}^{k} a_{l i} c_{l j}, \quad \delta_{\alpha \beta}=\widetilde{g}\left(\xi_{\alpha}^{\prime}{ }^{-}, \eta_{\beta}^{\prime}\right)=\sum_{\gamma=k+1}^{r} a_{\gamma \alpha} c_{\gamma \beta} .
$$

Thus we obtain $C_{-}={ }^{t} A_{+}^{-1}$ and $C_{+}={ }^{t} A_{-}^{-1}$. Furthermore, using $\widetilde{g}\left(\eta_{i}^{\prime}{ }^{+}, \eta_{\alpha}^{\prime}\right)=0$, we have

$$
0=\widetilde{g}\left(\eta_{i}^{\prime}, \eta_{\alpha}^{\prime}\right)=\sum_{j=1}^{k} b_{j \alpha} c_{j i}+\sum_{\beta=k+1}^{r} b_{\beta i} c_{\beta \alpha} .
$$

Hence, we consequently get $B_{-}=-A_{-}{ }^{t} B_{+}{ }^{t} A_{+}^{-1}$.

In case of $k(r-k) \neq 0$, for $(\xi ; \eta)$ and $\left(\xi^{\prime} ; \eta^{\prime}\right)$ of which is non-vanishing $M_{k, r-k}(\mathbb{R})$ valued function $B_{+}$on $U \cap U^{\prime}$, we see

$$
\operatorname{ltr}(T M, \xi) \neq \operatorname{ltr}\left(T M, \xi^{\prime}\right) \quad \text { on } \quad U \cap U^{\prime} .
$$

In the other hand, when $k(r-k)=0$, we can obtain the uniquely determined lightlike transversal bundle on $M$ as follows: 
Theorem 3.4. Let $(M, J, g)$ be a paracomplex r-lightlike submanifold of an almost parahermitian manifold $(\widetilde{M}, \widetilde{g}, \widetilde{J})$. If $\left(M, g, J, S(T M), S\left(T M^{\perp}\right)\right)$ satisfies

$$
\left.J\right|_{\operatorname{Rad}(T M)}=I_{\operatorname{Rad}(T M)} \quad \text { or }\left.\quad J\right|_{\operatorname{Rad}(T M)}=-I_{\operatorname{Rad}(T M)},
$$

then there uniquely exists the lightlike transversal vector bundle $\operatorname{ltr}(T M)$ over $M$ such that $\widetilde{J}$-invariant. Moreover, if $\left.J\right|_{\operatorname{Rad}(T M)}= \pm I_{\operatorname{Rad}(T M)}$, then $\left.\widetilde{J}\right|_{\operatorname{It}(T M)}=$ $\mp I_{\operatorname{ltr}(T M)}$.

Proof. By the assumption: $\left.J\right|_{\operatorname{Rad}(T M)}=I_{\operatorname{Rad}(T M)}$ or $\left.J\right|_{\operatorname{Rad}(T M)}=-I_{\operatorname{Rad}(T M)}$, we have $k(r-k)=0$. Then, from Lemma 3.2, it follows $B_{+}=O$ or/and $B_{-}=O$ for any $(\xi ; \eta)$ and $\left(\xi^{\prime} ; \eta^{\prime}\right)$ on $U$ and $U^{\prime}$ respectively. Therefore, we obtain

$$
\operatorname{ltr}(T M, \xi)=\operatorname{ltr}\left(T M, \xi^{\prime}\right) \quad \text { on } \quad U \cap U^{\prime} .
$$

Thus the lightlike transversal bundle is globally and uniquely determined on $M$. When $\left.J\right|_{\operatorname{Rad}(T M)}=I_{\operatorname{Rad}(T M)}$, since all signatures $\varepsilon_{i}(i=1, \ldots, k)$ in equations (3.1) in Theorem 3.2 are equal to +1 , we obtain $\left.\widetilde{J}\right|_{\operatorname{tr}(T M)}=-I_{\operatorname{ltr}(T M)}$. By a similar way, we can see $\left.\widetilde{J}\right|_{\operatorname{ltr}(T M)}=I_{\operatorname{ltr}(T M)}$, if $\left.J\right|_{\operatorname{Rad}(T M)}=-I_{\operatorname{Rad}(T M)}$. We have proved the theorem.

\section{PARACOMPleX LightLIKe SUbMANIFOLdS IN PARAKÄHLER MANIFOLDS}

In this section, we consider minimal lightlike submanifolds in semi-Riemannian manifolds. Sakaki [5] gives a definition of minimal lightlike submanifolds which is independent of the choice of the screen distribution and the screen transversal vector bundle as follows:

Definition 4.1. We say that a lightlike submanifold $(M, g)$ in a semi-Riemannian manifolds $(\widetilde{M}, \widetilde{g})$ is minimal if:

(a) $h(X, \xi)=0$ for any $X \in \Gamma(T M), \xi \in \Gamma(\operatorname{Rad}(T M))$, and

(b) trace $(h)=0$, where the trace is written with respect to $g$ restricted to $S(T M)$.

Remark 4.1. We also refer Bejan and Duggal [2] for another (original) definition of minimal lightlike submanifolds.

From now on, we take a parakähler manifold $(\widetilde{M}, \widetilde{g}, \widetilde{J})$ as the ambient space. Moreover, for a paracomplex $r$-lightlike submanifold $M$ of $(\widetilde{M}, \widetilde{g}, \widetilde{J})$, we choose vector bundles $S(T M), S\left(T M^{\perp}\right)$ and $\operatorname{ltr}(T M)$ are $\widetilde{J}$-invariant ones given in Theorem 3.3

Proposition 4.1. Let $\left(M, g, J, S(T M), S\left(T M^{\perp}\right)\right)$ be a paracomplex $r$-lightlike submanifold of a parakähler manifold $(\widetilde{M}, \widetilde{g}, \widetilde{J})$. Then $J$ is parallel with respect to the induced connection $\nabla$ and the second fundamental form $h$ satisfies $h(X, J Y)=$ $\widetilde{J} h(X, Y)$ for any $X, Y \in \Gamma(T M)$.

Proof. Taking a local basis $\xi=\left(\xi_{1}, \ldots, \xi_{r}\right)$ as in Lemma 3.1, we fix the $\widetilde{J}$-invariant lightlike transversal bundle $\operatorname{ltr}(T M)$. Then, for the induced connection $\nabla$, we have

$$
\widetilde{\nabla}_{X}(\widetilde{J} Y)=\left(\widetilde{\nabla}_{X} \widetilde{J}\right)(Y)+\widetilde{J}\left(\widetilde{\nabla}_{X} Y\right)=J\left(\nabla_{X} Y\right)+\widetilde{J}(h(X, Y)) .
$$

On the other hands, we get

$$
\widetilde{\nabla}_{X}(\widetilde{J} Y)=\nabla_{X}(J Y)+h(X, J Y)=\left(\nabla_{X} J\right)(Y)+J\left(\nabla_{X} Y\right)+h(X, J Y) .
$$


Because $T M$ and $\operatorname{tr}(T M)$ are $\widetilde{J}$-invariant, we obtain $\left(\nabla_{X} J\right)(Y)=0$ and $h(X, J Y)=$ $\widetilde{J} h(X, Y)$, which complete the proof.

The decomposition $\operatorname{tr}(T M)=S\left(T M^{\perp}\right) \oplus \operatorname{ltr}(T M)$ introduces

$$
h(X, Y)=h^{s}(X, Y)+h^{l}(X, Y) \text { for } X, Y \in T M,
$$

where $h^{s}$ (resp. $h^{l}$ ) is called the screen (resp. lightlike) second fundamental form of $M$. Since $S\left(T M^{\perp}\right)$ and $\operatorname{ltr}(T M)$ are $\widetilde{J}$-invariant, we obtain the following lemma:

Lemma 4.1. Under the above notations,

$$
h^{s}(X, J Y)=\widetilde{J} h^{s}(X, Y), \quad h^{l}(X, J Y)=\widetilde{J} h^{l}(X, Y) \quad \text { for } X, Y \in T M .
$$

Lemma 4.2. Let $\left(M, g, J, S(T M), S\left(T M^{\perp}\right)\right)$ be a paracomplex $r$-lightlike submanifold in a parakähler manifold $(\widetilde{M}, \widetilde{g}, \widetilde{J}) . \quad$ When $\left.J\right|_{\operatorname{Rad}(T M)}=I_{\operatorname{Rad}(T M)}($ resp. $\left.\left.J\right|_{\operatorname{Rad}(T M)}=-I_{\operatorname{Rad}(T M)}\right)$, we have for $X, Y \in \Gamma(T M)$,

$$
h^{l}(X, J Y)=-h^{l}(X, Y) \quad\left(\text { resp. } h^{l}(X, J Y)=h^{l}(X, Y)\right) .
$$

In particular, we obtain

$$
h^{l}\left(X^{+}, Y^{-}\right)=0 \text { for } X^{+} \in \Gamma\left(T M^{+}\right) \text {and } Y^{-} \in \Gamma\left(T M^{-}\right) .
$$

Proof. When $\left.J\right|_{\operatorname{Rad}(T M)}=I_{\operatorname{Rad}(T M)}$, a local basis $\eta=\left(\eta_{1}, \ldots, \eta_{r}\right)$ of $\operatorname{ltr}(T M)$ as in Theorem 3.2 satisfy $\widetilde{J} \eta_{i}=-\eta_{i}(i=1, \ldots, r)$. Writing the lightlike second fundamental form $h^{l}$ as follows

$$
h^{l}(X, Y)=\sum_{i=1}^{r} h_{i}^{l}(X, Y) \eta_{i}
$$

we have

$$
\begin{aligned}
h^{l}(X, J Y) & =\widetilde{J} h^{l}(X, Y)=\widetilde{J}\left(\sum_{i=1}^{r} h_{i}^{l}(X, Y) \eta_{i}\right) \\
& =\sum_{i=1}^{r} h_{i}^{l}(X, Y) \widetilde{J} \eta_{i}=-\sum_{i=1}^{r} h_{i}^{l}(X, Y) \eta_{i}=-h^{l}(X, Y) .
\end{aligned}
$$

We can similarly prove, in the case of $\left.J\right|_{\operatorname{Rad}(T M)}=-I_{\operatorname{Rad}(T M)}$.

We call a $r$-lightlike submanifold $(M, g)$ co-isotropic if $r=\operatorname{codim} M$. Then we recognize $S\left(T M^{\perp}\right)$ as the zero vector bundle, hence $h^{s}=0$.

Theorem 4.1. Let $(M, g, J, S(T M))$ be a co-isotropic paracomplex submanifold of a parakähler manifold $(\widetilde{M}, \widetilde{g}, \widetilde{J})$. If $\left.J\right|_{\operatorname{Rad}(T M)}= \pm I_{\operatorname{Rad}(T M)}$, then $(M, g)$ is minimal in the sense of Definition 4.1.

Proof. Without a loss of generalities, we can assume $\left.J\right|_{\operatorname{Rad}(T M)}=I_{\operatorname{Rad}(T M)}$. By the assumption, $S(T M)$ is a parahermitian vector bundle. Thus, we can take a local orthonormal basis $X_{1}, X_{2}, \ldots, X_{2 s-1}, X_{2 s}$ of $S(T M)$ such that $g\left(X_{i}, X_{j}\right)=(-1)^{i} \delta_{i j}$ for $i, j=1, \ldots, 2 s$, and $X_{2 i}=J\left(X_{2 i-1}\right)$ for $i=1, \ldots, s$, where $\operatorname{rank}(S(T M))=2 s$ 
and index $(S(T M))=s$. Then we obtain

$$
\begin{aligned}
\operatorname{trace}(h) & =\sum_{i=1}^{s}\left(-h\left(X_{2 i-1}, X_{2 i-1}\right)+h\left(X_{2 i}, X_{2 i}\right)\right) \\
& =\sum_{i=1}^{s}\left(-h\left(X_{2 i-1}, X_{2 i-1}\right)+h\left(J\left(X_{2 i-1}\right), J\left(X_{2 i-1}\right)\right)\right) \\
& =\sum_{i=1}^{s}\left(-h\left(X_{2 i-1}, X_{2 i-1}\right)+h\left(X_{2 i-1}, X_{2 i-1}\right)\right)=0 .
\end{aligned}
$$

Hence the condition (b) in Definition 4.1 holds.

Since $(M, g)$ is co-isotropic, we have $h^{s}=0$. In general, we can see that the lightlike second fundamental form $h^{l}$ is vanishing on $\operatorname{Rad}(T M)$, from $[3$, p.157, Proposition. 2.2] or [4, p.199. Proposition. 5.1.3]. According the decomposition: $T M=S(T M) \oplus \operatorname{Rad}(T M)$, we decompose $X \in T M$ as $X=X_{S}+X_{R}$. Moreover, for any $X \in S(T M)$, we decompose $X$ as $X=X^{+}+X^{-}$, where $J\left(X^{ \pm}\right)= \pm X$. Then we obtain

$$
\begin{aligned}
h(X, \xi) & =h^{l}(X, \xi)=h^{l}\left(X_{S}+X_{R}, \xi\right)=h^{l}\left(X_{S}, \xi\right)+h^{l}\left(X_{R}, \xi\right)=h^{l}\left(X_{S}, \xi\right) \\
& =h^{l}\left(X_{S}^{+}+X_{S}^{-}, \xi\right)=h^{l}\left(X_{S}^{+}, \xi\right)+h^{l}\left(X_{S}^{-}, \xi\right) .
\end{aligned}
$$

By virtue of Lemma 4.2 and $\xi \in \operatorname{Rad}(T M)=\operatorname{Rad}(T M)^{+}, h^{l}\left(X_{S}^{-}, \xi\right)=0$. From Lemma 4.2 and $\left.J\right|_{\operatorname{Rad}(T M)}=I_{\operatorname{Rad}(T M)}$ again, we have $h^{l}\left(X_{S}^{+}, \xi\right)=h^{l}\left(X_{S}^{+}, J \xi\right)=$ $-h^{l}\left(X_{S}^{+}, \xi\right)$, thus $h^{l}\left(X_{S}^{+}, \xi\right)=0$. Hence the condition (a) in Definition 4.1 holds.

Remark 4.2. Sakaki gives examples of minimal lightlike submanifolds in [5, Theorem 5.1]. The examples satisfy the conditions as in Theorem 4.1.

From Corollary 3.1 and Theorem 4.1, we obtain

Corollary 4.1. Any paracomplex lightlike hypersurfaces in a parakähler manifold are 1-lightlike and minimal.

Acknowledgement. The author would like to express his sincere gratitude to Professor Naoto Abe and Professor Makoto Sakaki for their valuable advices.

\section{REFERENCES}

[1] Al-Aqeel, A. and Bejancu, A., On the geometry of paracomplex submanifolds, Demonstratio Math. 34 (2001), No. 4, 919-932.

[2] Bejan C.L. and Duggal, K.L., Global lightlike manifolds and harmonicity, Kodai Math. J., 28 (2005), 131-145.

[3] Duggal, K. L. and Bejancu, A., Lightlike submanifolds of semi-Riemannian manifolds and applications, Kluwer Academic Publishers, Dordrecht, 1996.

[4] Duggal, K. L. and Sahin, B., Differential geometry of lightlike submanifolds, Birkhäuser Verlag, Basel, 2010.

[5] Sakaki, M., On the definition of minimal lightlike submanifolds, Int. Electron. J. Geom. 3 (2010), No. 1, 16-23.

Department of mathematics, Faculty of science, Tokyo University of SCience, 1-3 Kagurazaka Shinjuku-Ku, Tokyo, 162-8601, JaPan

E-mail address: kmiura@rs.tus.ac.jp 